\title{
Muscle recruitment patterns during the prone leg extension
} Gregory J Lehman*1, Duane Lennon², Brian Tresidder ${ }^{2}$, Ben Rayfield ${ }^{2}$ and Michael Poschar ${ }^{2}$

\author{
Address: ${ }^{1}$ Department of Graduate Studies and Research, Canadian Memorial Chiropractic College, 1900 Bayview Ave, Toronto, ON, Canada and \\ ${ }^{2}$ Undergraduate Department, Canadian Memorial Chiropractic College, 1900 Bayview Ave, Toronto, ON, Canada \\ Email: Gregory J Lehman* - glehman@cmcc.ca; Duane Lennon - duane_lennon@hotmail.com; Brian Tresidder - rayfield@hotmail.com; \\ Ben Rayfield - benrayfield@hotmail.com; Michael Poschar - poshdc@hotmail.com \\ * Corresponding author
}

Published: 10 February 2004

BMC Musculoskeletal Disorders 2004, 5:3
Received: 10 November 2003

Accepted: 10 February 2004

This article is available from: http://www.biomedcentral.com/147/-2474/5/3

(C) 2004 Lehman et al; licensee BioMed Central Ltd. This is an Open Access article: verbatim copying and redistribution of this article are permitted in all media for any purpose, provided this notice is preserved along with the article's original URL.

\begin{abstract}
Background: The prone leg extension (PLE) is a clinical test used to evaluate the function of the lumbopelvis. It has been theorized that a normal and consistent pattern of muscle activation exists. Previous research has found two contradictory patterns of muscle activation during PLE in normal individuals. One study shows an almost simultaneous activation of the lower erector spinae and hamstring muscle group with a delayed activation of the gluteus maximus, while the second describes the order of activation being ipsilateral erector spinae (to the leg being extended), hamstrings, contralateral erector spinae and gluteus maximus. Due to the different conclusions from these two studies and the lack of quantified muscle onset times, expressed in absolute time this study attempted to quantify the muscle onset times (in milliseconds) during the prone leg extension, while noting if a consistent order of activation exists and whether a timing relationship also exists between the gluteus maximus and contralateral latissimus dorsi.
\end{abstract}

Methods: 10 asymptomatic males (Average height: $175.2 \mathrm{~cm}$ (SD 6.5), Average Weight $75.9 \mathrm{~kg}$ (SD 6.5), Average Age: 27.I (SD I.28)) and 4 asymptomatic females (Average height 164.5 (SD 2.9), weight: 56.2 (SD 8.9), Average Age: 25 (SD I)) performed the prone leg extension task while the myoelectric signal was recorded from the bilateral lower erector spinae, gluteus maximus and hamstring muscle groups. Activation onsets were determined from the rectified EMG signal relative to the onset of the hamstrings muscle group.

Results: No consistent recruitment patterns were detected for prone leg extension among the hamstring muscle group and the erector spinae. However, a consistent delay in the Gluteus Maximus firing of approximately $370 \mathrm{~ms}$ after the first muscle activated was found. Five out of 14 asymptomatic subjects showed a delay in gluteus maximus firing exceeding the average delay found in previous research of subjects considered to have a dysfunctional firing pattern.

Conclusion: A consistent pattern of activation was not found. Variability was seen across subjects. These findings suggest the PLE is not sufficient for a diagnostic test due to the notable physiological variation. An overlap between normal and potentially abnormal activation patterns may exist. 


\section{Background}

The prone leg extension test is commonly used in the evaluation of lumbopelvic function. It has been theorized that the activation of muscles during a prone leg extension (PLE) simulates the muscle recruitment pattern of hip extension during gait. The theory suggests that the temporal activation of the posterior muscle groups should occur in the following order (right PLE exercise): right gluteus maximus, right hamstring, left lumbar erector spinae, right lumbar erector spinae, left thoracolumbar erector spinae and lastly right thoracolumbar erector spinae [1].

According to this theory, differences in temporal recruitment patterns decrease the stability of the pelvis during gait and thus hinder the body's mechanical efficiency. It is the clinical belief that a delay in the gluteus maximus recruitment is a dysfunctional pattern of muscular recruitment. The theory concludes that the poorest recruitment pattern during the PLE occurs when the ipsilateral erector spinae and even the shoulder girdle muscles initiate the movement. The gluteus muscle activation is delayed and hip extension is achieved by hamstring muscle activation, forward pelvic tilt and hyperlordosis of the lumbar spine. Sahrman [2] has suggested that if the hamstrings are dominant because of inhibition of the gluteus maximus an anterior shear of the trochanter can be palpated during the prone leg extension. Poor gluteus maximus strength and activation is postulated to decrease the efficiency of gait $[3,4]$.

Bullock-Saxton et al [5] and Vogt and Banzer [6] suggests that there is a consistent pattern of muscle activation during prone leg extension (although they disagree on the order of firing) and imply that there is a fixed motor program in normal subjects. Bullock-Saxton et al concluded that in pain free subjects muscle onset times were "almost simultaneous". A muscle time span (in seconds) between the activation of the first muscle to the muscle to be last activated was calculated. For the control group the average time span was .306 seconds. They stated that the Hamstrings typically were the first recruited. Furthermore, Bullock-Saxton et al found a lack of consistency and a higher degree of variability within subjects who had sustained previous ankle injury [5].

Vogt and Banzer [6] evaluated the muscle onset times during the PLE in pain free subjects finding statistically different activation onset times for the muscles studied. The authors found that the muscles studied fired in the following order: ipsilateral lumbar erector spinae, semitendinosis, contralateral lumbar erector spinae, tensor facia latae and gluteus maximus. However, when the means and standard deviations (expressed as a percentage of the movement cycle) are compared for the onsets of the ipsilateral erector spinae $($ mean $=13.91, \mathrm{SD}=10.97)$, contral- ateral erector spinae $($ mean $=17.27, \mathrm{SD}=12.86)$ and hamstrings $($ mean $=17.61, \mathrm{SD}=13.04)$ there is a great deal of overlap and they occur very close in time (amount expressed in seconds not known). This proximity in time may be identical to what the Bullock-Saxton study found but described as "almost simultaneous". Again, with the large overlap in the Vogt and Banzer [6] study, it is possible that some of the muscles came on in a different order even though statistically a significant difference between the muscle onsets was found.

The differences between the conclusions may have been due to collecting similar data, analyzing it slightly differently and subsequently finding a different conclusion. One similarity between the two studies is that the gluteus maximus is consistently the last muscle to become active. A functional and anatomical relationship has also been described between the gluteus maximus and the contralateral latissimus dorsi which is theorized to ensure stability of the SI joint during gait and movement [7]. Due to this relationship the muscle activation of the contralateral latissimus dorsi was also studied during the prone leg extension in the current study.

The performance of the prone leg extension during a physical exam is based on the belief that a "normal "pattern of muscle activation occurs. If variability in muscle recruitment times occurs across patients then the use of this test in identifying dysfunction may be limited. Due to the different conclusions from two previous studies and the lack of quantified muscle onset times, expressed in absolute time (milliseconds) this study had two objectives.

1. Develop a database of muscle onset times (in milliseconds) for the posterior muscle groups during the prone leg extension, while noting if a consistent order of activation exists and whether a timing relationship also exists between the gluteus maximus and contralateral latissimus dorsi.

2. Express the muscle onset times in relation to the onset of hamstring muscle activity to alleviate the need for timing equipment to determine onset of movement.

\section{Methods}

Ten males (Average height: $175.2 \mathrm{~cm}$ (SD 6.5), Average Weight $75.9 \mathrm{~kg}$ (SD 6.5), Average Age: 27.1(SD 1.28) and 4 females (Average height 164.5 (SD 2.9), weight: $56.2 \mathrm{~kg}$ (SD 8.9), Average Age: 25 (SD 1)) with no history of lumbar, sacroiliac or lower limb injury within the past year were recruited from a convenience sample of CMCC students. Subjects were excluded if they had previous lumbar surgery, spondylopathies or arthritic disorders. Subjects read and signed an information and consent form approved by the Internal Review Board of CMCC. 


\section{Study Protocol}

The muscle activity of the left latissimus dorsi, right gluteus maximus, bilateral lower erector spinae, and right hamstring muscle groups was recorded during right prone leg extension.

\section{Data Collection Hardware Characteristics}

Disposable bipolar Ag-AgCl disc surface electrodes with a diameter of one $\mathrm{cm}$ was adhered bilaterally over the five muscle groups with a centre to centre spacing of $2.5 \mathrm{~cm}$. Raw EMG was amplified between 1000 and 20,000 times depending on the subject. The amplifier has a CMRR of 10,000:1 (Bortec EMG, Calgary AB, Canada). Raw EMG was band pass filtered (10 and $1000 \mathrm{~Hz})$ and A/D converted at $2000 \mathrm{~Hz}$ using a National Instruments data acquisition system.

\section{Exercise tasks}

The prone leg extension exercise was performed five times for each subject. The task required the subject to lie prone while EMG activity was collected for 5 seconds. The subject then extended their straight right leg approximately six inches off the table. The leg was held isometrically for 3 seconds then lowered to the table.

\section{EMG Processing}

For each repetition, and every subject the data underwent the same procedure. The aim of the processing for this study was to determine the order and timing of muscle activation. To determine muscle timing, it is necessary to determine when a muscle is considered active or "on". A muscle was considered "on" when the level of muscle was greater than $10 \%$ of the peak muscle activity during the prone leg extension. This method of determining muscle onset was used in the previous study by Bullock-Saxton et al [5]. The order of activation can then be determined by classifying each muscle as "on" when its level of activity exceeds that of its predetermined threshold. Muscle activation time was referenced to the time of activation of the hamstring muscle. For example, positive values (ms) occurred when muscle onset was before activation of the hamstrings, and negative values indicated that muscle activation occurred after the onset of the hamstring muscle. The onset of muscle activity was determined for each muscle during each repetition of the prone leg lift.

\section{Data \& Statistical Analysis}

The purpose of the analysis was to determine if a consistent order of activation existed across subjects. This was a simple qualitative analysis to assess whether subjects recruit the posterior muscles in the same order. The average of the muscle onset times for the 5 repetitions for each of the five muscles was calculated for each subject. The order of activation was then determined for each subject and a qualitative assessment of how many subjects have the same recruitment order was assessed. The group average and standard deviation of muscle onset times for each muscle was also calculated. A Kruskal-Wallis test (nonparametric ANOVA) with a post hoc Dunn test was used to determine if the average onset times were different for each muscle group.

\section{Results}

\begin{tabular}{|c|c|c|c|c|c|c|}
\hline Subject \# & Right ES (I) & Left ES (2) & GM (3) & Hamstring (4) & Time Span (ms) & Order \\
\hline I & 29.3 & 8.8 & -598.7 & 0 & 628 & 1243 \\
\hline 2 & -173.5 & 124.3 & -96.8 & 0 & 173 & 2431 \\
\hline 3 & -13.8 & -125.5 & -676.5 & 0 & 676 & 4123 \\
\hline 4 & 12 & -1.3 & -70.75 & 0 & 70 & 1423 \\
\hline 5 & $-|76|$. & -126 & -510.7 & 0 & 510 & 4213 \\
\hline 6 & -111.3 & $-|5|$ & -226.6 & 0 & 226 & 4123 \\
\hline 7 & 22 & 14.6 & -107.3 & 0 & 107 & 2143 \\
\hline 8 & 59 & -.25 & -102 & 0 & 102 & 1423 \\
\hline 9 & -15.25 & 23.12 & $-|3|$ & 0 & $|3|$ & 2413 \\
\hline 10 & -46.7 & -41.25 & -269.5 & 0 & 269 & 4213 \\
\hline 11 & -227.5 & 5 & -611.25 & 0 & 611 & 2413 \\
\hline 12 & -2.625 & 34.5 & -407.6 & 0 & 407 & 2413 \\
\hline 13 & 223.2 & 48.25 & -469.12 & 0 & 692 & 1243 \\
\hline 14 & -42.6 & 116.8 & -576.7 & 0 & 576 & 2413 \\
\hline Mean & -33.14 & -5.01 & -346 & & 370.23 & \\
\hline S.D & $1 \mid 4.06$ & 82.19 & 225.87 & & 230.2 & \\
\hline
\end{tabular}

Represents EMG onset times relative to hamstring firing. ES = Erector Spinae, GM = Gluteus Maximus, $m s=$ milliseonds and SD $=$ Standard Deviation. 
Table 1 shows the EMG onset times for each of the muscle groups examined and the order of recruitment for these muscle groups for the 14 subjects. The onset timing of the Gluteus Maximus was significantly delayed ( $<$ <.05) compared with all other muscles. The remaining muscles activation times were not statistically significantly different from each other. The timing of activation for the latissimus dorsi muscle was not included as the muscle did not become active for the majority of the trials. For the remainder of the muscles, the EMG onset times were recorded relative to hamstring firing. In $6 / 14$ subjects the first muscle to become active during the prone leg extension was the contralateral erector spinae muscles. In 8/14 subjects the contralateral lumbar erector fired before hamstrings. In 4/14 subjects the ipsilateral lumbar erector was the first muscle to fire and in 5/14 subjects the ipsilateral lumbar erector was recruited prior to the initiation of hamstring movement. In $4 / 14$ subjects the hamstrings were the first muscle group activated and in 13/14 subjects the gluteus maximus was the last muscle active during the prone leg extension.

When muscle onset times were averaged, with the hamstring muscle acting as the relative starting point at $0 \mathrm{~ms}$, the contralateral erector spinae became active $5.01 \mathrm{~ms}$ later, followed by the contralateral erector spinae 33.14 ms hence and last to fire was the gluteus maximus, 346.8 ms after the onset of the hamstrings. While averaging the onset times appears to indicate that the hamstring muscle becomes active first, the qualitative data explains that this occurred only in four subjects, however, due to the large range in onset times the average can be skewed to suggest an order of activation which is not typical.

The average time span between the activation of the first muscle to the last muscle was $370.2 \mathrm{~ms}$. Five out of 14 subjects exceeded the average time span of $527 \mathrm{~ms}$ found in subjects with ankle sprains in the study by Bullock-Saxton et al which was considered to be indicative of an aberrant firing pattern.

\section{Discussion}

The analysis of EMG data in this study shows a large variability in muscle activation order between subjects, with no consistent firing pattern in asymptomatic individuals. Different muscles came on first and on average 3 muscles fired within $33 \mathrm{~ms}$ of each other, a statistically non-significant difference. In this current study the only consistent finding between subjects was that $13 / 14$ subjects fired the gluteus maximus last. This agrees with the results of the two previous studies in asymptomatic individuals.

Not only are the findings of this current study contrary to the proposed pattern of muscle recruitment [4,1] and conclusions from previous studies, it is also questions the idea proposed by Bullock-Saxton et al., $[5,8]$ stating that delayed gluteus maximus firing is abnormal. Bullock-Saxton et al., found that those subjects with previous ankle sprains had an increased activation time span between the first and last muscle to become activated of $527 \mathrm{~ms}$. In our study, 5 of the 14 asymptomatic subjects had an activation time span greater than this value. This suggests that the prone leg extension is not sufficient for a diagnostic test due to the notable physiological variation and the probable expected overlap between normal and potentially abnormal activation patterns i.e. delay gluteus maximus firing.

While Janda [1,3,4] and Singer [9] both advocate the assessment of muscle firing order during active prone leg extension as part of the examination of spinal dysfunction, the timing of activation of 3 muscles appear to be within $30 \mathrm{~ms}$ of each other which questions whether one can assess these activation patterns without surface EMG. Even if a delay of approximately $200 \mathrm{~ms}$ in the gluteus maximus is indicative of pathology can this delay be detected without functional testing that incorporates EMG i.e. muscle palpation? Functional testing procedures should be validated further before dysfunctional recruitment patterns in symptomatic individuals can truly be identified. The inconsistencies in the firing pattern during the prone leg extension suggest the need to create and evaluate other methods to objectively assess neuromuscular functional control.

One large limitation may be our inability to identify what is truly an abnormal pattern of muscular activation. While the participants included in this study had no current symptoms they may still have dysfunctional motor activation patterns which have not presented symptomatically. Future work should look at the relationship between activation patterns and the onset of future dysfunction. It should also be noted that the PLE test is also used to assess the movement kinematics of patients. This paper only investigated muscle onset timing and did not assess movement kinematics. The PLE test may still be a valid test for assessing movement dysfunction, however, no work has been done to assess this possibility.

\section{Conclusion}

This study found no consistent order of activation for the biceps femoris, contralateral erector spinae and ipsilateral erector spinae during prone leg extension. Consistently, the gluteus maximus is the last to become active with onset timing ranging from $70 \mathrm{~ms}$ to $676 \mathrm{~ms}$. The latissimus dorsi does not appear to become activated, nor activated with any temporal relationship to its contralateral gluteus maximus muscle. 


\section{Competing Interests}

None declared.

\section{Author Contributions}

GL: conception, design, data collection, data analysis, manuscript preparation

BR, MP, DL, BT: design, data collection, manuscript preparation

\section{References}

I. Janda V: Muscle spasm - a proposed procedure for differential diagnosis. Journal of Manual Medicine I99|, 6(4): |36-I39.

2. Sahrman S: Diagnosis and Treatment of Movement Impairment Syndromes. Ist edition. Mosby Inc; 2002.

3. Janda V: Evaluation of Muscular Imbalance (Ch.6). Rehabillitation of the Spine: A Practitioner's Manual I 1996:97-III.

4. Janda V: Treatment of chronic low back pain. Journal of Manual Medicine 1992, 6(5): 166-168.

5. Bullock-Saxton JE, Janda V, Bullock MI: The influence of ankle spraininjury on muscle activation during hip extension. Int J Sports Med 1994, I 5(6):330-4.

6. Vogt L, Banzer W: Dynamic testing of the motor stereotype in prone hip extension from neutral position. Clin Biomech 1997, I 2(2): I22- I 27.

7. Mooney V, Pozos R, Vleeming A, Gulick J, Swenski D: Exercise treatment for sacroiliac pain. Orthopedics 200I, 24(I):29-32.

8. Bullock-Saxton JE, Janda $\mathrm{V}$, Bullock Ml: Reflex activation of gluteal muscles in walking. Spine 1993, 18(6):704-708.

9. Singer KP: $\mathbf{A}$ new musculoskeletal assessment in a student population. J Orthop Sports Phys Ther 1986, 8:34-4I.

\section{Pre-publication history}

The pre-publication history for this paper can be accessed here:

http://www.biomedcentral.com/1471-2474/5/3/prepub

Publish with Bio Med Central and every scientist can read your work free of charge

"BioMed Central will be the most significant development for disseminating the results of biomedical research in our lifetime."

Sir Paul Nurse, Cancer Research UK

Your research papers will be:

- available free of charge to the entire biomedical community

- peer reviewed and published immediately upon acceptance

- cited in PubMed and archived on PubMed Central

- yours - you keep the copyright 